\title{
A New Class of Polymeric Anti-Allergen Nasal Barrier Film Solution for the Treatment of Allergic Rhinitis
}

Lea Shrivastava ${ }^{1}$, Hendrik Schütte ${ }^{2}$, Pawan Malik ${ }^{3}$ and Ravi Shrivastava ${ }^{1^{\star}}$

${ }^{1}$ Vitro-Bio Research Institute, Issoire, France

${ }^{2}$ Strathos Pharma Group, Bad Ems, Germany

${ }^{3}$ Wockhardt Bio AG, Dubai, UAE

*Corresponding author: Ravi Shrivastava, Vitro-Bio Research Institute, 63500 Issoire, France, Tel: +33 (0)4 73550505 ; Fax: +33 (0)4 735500 11; E-mail: rs@vitrobio.com

Received date: July 11, 2017; Accepted date: August 18, 2017; Published date: August 26, 2017

Copyright: $\odot 2017$ Shrivastava L, et al. This is an open-access article distributed under the terms of the Creative Commons Attribution License, which permits unrestricted use, distribution, and reproduction in any medium, provided the original author and source are credited.

\begin{abstract}
Objective: Allergic rhinitis (AR) is oversensitivity of nasal mucosa to allergen exposure leading to IgE-mediated nasal mucosal inflammation and cellular destruction. Applying any chemical drug over the nasal mucosa may provide symptomatic relief but at the same time the presence of chemicals, such as cortisones and antihistaminic, hampers mucosa repair and, in consequence, cellular defense functions. Therefore, cleaning, avoiding allergen exposure, and protecting nasal mucosa from contact with allergens with a cell-friendly mechanical device, could be an effective remedy to treat allergic rhinitis.
\end{abstract}

Methods: A double blind, randomized, placebo controlled, multicenter clinical trial was conducted in patients suffering from severe AR. 15 patients were treated with saline as comparator product (CP) versus 31 treated with the test product (TP). The test product contained a natural gum-glycerol solution (VB-Gy) which was rendered filmogen using inert natural polymers (Allercyanidin-H) and termed as VB-Gy-Allercyanidin-H formula. The products (15 ml sprays) were applied topically over the nasal mucosa, 3-4 times a day over a period of 3 weeks. Total, reflective and instantaneous nasal symptom scores for rhinorrhea, nasal discharge, sneezing, and itching, as well as ocular scores (itching, tearing, redness) and rescue medicine use scores were evaluated daily during weeks -1 to +3 employing a 0 (no symptoms) to 3 (severe symptoms) scoring scale. Rhino-conjunctivitis quality of life (RQLQ) questionnaires were completed at the start and at the end of the study. Saline solution (CP) was used identically to the TP. Mean weekly results in CP and TP groups were compared with the scores at the start of treatment (baseline) and between the two groups.

Results: CP was found to reduce only slightly the symptomatic manifestation of allergic rhinitis. The mean reduction compared to baseline at the end of weeks 1,2 , and 3 was respectively $11.7 \%, 13.6 \%$ and $15.1 \%$ for total nasal symptom scores (rTNSS); $9.9 \%, 14.5 \%$, and $15.8 \%$ for total ocular symptom scores (rTOSS); and $4.97 \%$, $8.45 \%$, and $10.94 \%$ for pre-dose instantaneous total ocular symptom scores (am-iTOSS, p: Not Significant: NS). During the same period, compared to CP scores, the reduction in TP group was higher by $37.7 \%, 58.4 \%$, and $73.5 \%$ for rTNSS; $38.3 \%, 54.6 \%$, and $64.1 \%$ for rTOSS and $29.84 \%, 48.91 \%$, and $59.77 \%$ for am-iTOSS ( $p<0.05$ for all parameters vs. CP at the same time points). The rhinoconjunctivitis quality of life questionnaire (RQLQ), measured using standard established questionnaire, was improved by $50.28 \%$ in TP group compared to $22.85 \%$ in CP group. During the study period, at least one rescue medicine was used by $80 \%$ patients in CP group compared to only $29 \%$ in TP group. Both products were well tolerated and induced no undesired effects.

Conclusion: In the absence of any cell-friendly, safe, and multi-target treatment for allergic rhinitis, using a mechanically acting, filmogen barrier solution capable of blocking new allergen contact and minimizing the concentration of immune cells on the nasal surface represents a simple but highly effective approach for the treatment of common allergic rhinitis.

Keywords: Clinical; Filmogen; Osmotically active; Allergic rhinitis; Treatment

\section{Introduction}

Allergic rhinitis (AR) is manifested as sneezing, rhinorrhea, nasal itching, congestion, and ocular discharge, which are allergen-driven nasal mucosa inflammatory symptoms modulated by immunoglobulin E [1]. Epidemiological studies have indicated that prevalence of AR continues to increase, and it has been a worldwide health problem that places a significant healthcare burden on individuals and society [2,3]. According to the severity, allergic rhinitis can be classified as mild, moderate or severe, and according to the duration of inflammatory reaction as acute ( $<6$ weeks) or chronic ( $>6$ weeks) [4]. AR topical immunological reactions involve numerous inflammatory cells, including mast cells, CD4-positive T cells, B cells, macrophages, and eosinophils, which infiltrate the nasal lining upon exposure to an inciting allergen, most commonly airborne dust, mite fecal particles, cockroach residues, animal dander, molds, and pollens. The $\mathrm{T}$ cells infiltrating the nasal mucosa are predominantly $\mathrm{T}$ helper (Th) 2 in 
Page 2 of 9

nature, and release cytokines (e.g., interleukin [IL]-3, IL-4, IL-5, IL-9, and IL-13) that promote immunoglobulin $\mathrm{E}$ (IgE) production by plasma cells [5]. IgE production, in turn, triggers the release of mediators, such as histamine and leukotrienes, that dilate blood vessels, increase vascular permeability, and cause itching, rhinorrhea and other symptoms of AR. If the allergen attack is not stopped, the inflammatory cascade continues, with chronic presence of proinflammatory cytokines and histamine over the nasal mucosa leading to chronic AR [6,7].

The ideal solution is to avoid exposing the nasal mucosa to allergic reaction-triggering allergens, but this is not always possible to achieve. The second option should be to protect the nasal mucosa with a bandage that can allow easy respiration and normal nasal mucosa functions while preventing nasal mucosa - allergen contact. Such a bandage should also minimize the concentration of free-floating immune cells and histamine on the nasal mucosa to reduce inflammation and allow cellular repair for chronic relief. Unfortunately, except for frequent nasal washes with normal saline, all other currently available therapeutic options such as oral antihistamines, intranasal corticosteroids, leukotriene receptor antagonists, decongestants and oral corticosteroids, are mono-target and symptomatic with limited efficacy [8].

Natural and synthetic polymers have already been used recently to improve polyol filmogenicity, in pharmaceutical industries as coating agents, and in medical devices $[9,10]$. We postulated that applying a transparent, filmogen liquid bandage, capable of protecting and cleaning the nasal surface without exacerbating rhinorrhea, should fulfill these basic ideal treatment requirements. Cleaning and protecting the nasal mucosa should also help repair cellular damage and improve local defenses. The efficacy and safety of such a filmogen bandage (VB-Gy-allercyanidin-H) was evaluated in patients suffering from chronic AR.

\section{Materials and Methods}

\section{Test product}

The test product (TP) comprised a synergistic association of acacia and xanthan gums with glycerol in water as a viscous solution (VBGy). As VB-Gy alone would get diluted with the hypotonic liquid outflow it generates, it was improved to become filmogen, flexible and more resistant to dilution by adding a small quantity of allercyanidins, containing a specific association of natural polymeric ingredients such as plant extracts, natural gums, plant cellulose, and hydrophobic essential oils as described by Shrivastava et al. [11]. This full filmogen solution was termed "VB-Gy-Allercyanidin- $\mathrm{H}$ " formula. These ingredients were selected based on their glycerol film retention properties from non-irritant plants such as Camellia sinensis leaves, Panax ginseng roots, Calendula officinalis flowers, Curcuma longa roots, Acacia catechu gum, Hedera helix leaves, proanthocyanin rich fruits, or hydrophobic essential oils.

The aim was to employ slight hypertonic properties of glycerol to detach and drain all the immunogenic cells and histamine molecules from the surface and to protect the nasal mucosa using a long lasting filmogen barrier.

\section{Study sponsor}

Naturveda Research Institute, ZAC de Lavaur 63500 Issoire, France who holds the patent, sponsored the trial. The study was conducted between 02-2016 and 09-2016.

\section{Study organizer}

The clinical part of this research was conducted at Nexus Clinical Research Center in India, affiliated to Nexus Clinical Research LLC, USA. The protocol and study design were approved by the Institutional Ethical Committee of India - Rajiv Gandhi Institute of medical sciences (EC Registration $\mathrm{N}^{\circ}$ ECR/492/Inst/AP/2013, dated 05/12/2013) and the trial was conducted following the ICH-GCP guidelines as per the declaration of Helsinki concerning ethical principles for medical research involving human subjects.

\section{Study design}

The study was comparative, randomized, double-blind, parallel group, observational clinical trial study for the treatment of allergic rhinitis in male and female patients, aged between 08 and 65 years. The aim of the study was to compare the efficacy and safety of the test product (TP): VB-Gy-Allercyanidin-H liquid formula using physiological saline solution, as comparator product $(\mathrm{CP})$.

\section{Study rationale}

The objective was to collect results on at least 36 patients in TP group and 18 in $\mathrm{CP}$ group to obtain statistically significant data. It was decided to include more patients in the TP group (in a 2:1 ratio) as use of saline solution for the treatment of AR is well documented and its efficacy is well known. The doses were selected based on dose range findings from a previous pilot observational study where treatment was administered 3 times per day for 14 days without any side effects. $0.9 \% \mathrm{NaCl}$ saline spray was chosen as comparator product because saline solution is commonly used for symptomatic relief of AR and it is applied identically to the TP. One of the secondary objectives of the study was to quantify rescue medicine requirements in $\mathrm{CP} v s$. TP group as the use of rescue medication (RM), such as anti-histaminic drugs, is common for the symptomatic relief of AR. The Rhinoconjunctivitis quality of life (RQLQ) parameters were evaluated as described by Filanowicz et al. [12] to analyze overall product efficacy. Although this product can be used by patients suffering from other diseases, pregnant women, or young children, it was decided to exclude patients which may affect study outcome, for product efficacy comparison.

\section{Inclusion and exclusion criteria}

At the time of recruitment at the study centers, patients were examined physically and patient's medical, surgical, and allergic history was checked and recorded. Vital signs such as blood pressure, pulse rate, and respiratory rate were recorded. Patients not suffering from any serious pathology and having a history of at least 2-year acute seasonal or perennial hay fever AR symptoms were then examined for enrollment in the study. The main inclusion criteria comprised: positive for seasonal skin prick test, ready to abstain from all antiallergic medication or any other treatment which may affect study outcome (except in the case of strict necessity where such treatment was prescribed by the clinical investigator), patients having mean daily reflective Total Nasal Symptom Score (rTNSS) of $\geq 3$ on a scale of 6 
and mean daily Reflective Ocular Symptom Score (rTOSS) of $\geq 2$ on a scale of 4 for the previous 7 days (day -6 to 0 ). Key exclusion criteria included: hypersensitivity to any of the investigational products components, respiratory diseases, or being under any treatment which may affect the study outcome.

\section{Randomization}

After screening, patients satisfying all the inclusion criteria were enrolled and randomly allocated, in a 2:1 ratio, to either test product (TP) or comparator product (CP) groups. Randomization was performed using SAS Version 9.1.3 following a randomization schedule. Block Randomization methodology was employed for generating the list. Within the block the treatments were distributed in the ratio of 2:1. Each patient received a unique screening identification number, randomization code, and enrollment identification number and a personal diary for recording.

\section{Product presentation and administration}

TPs and CPs were supplied by Naturveda R\&D institute, France (Issoire) and were presented identically (15 $\mathrm{ml}$ spray containing a slightly viscous and colorless liquid) except for the product code and the batch number. The TP contained VB-Gy-Allercyanidin-H solution while $\mathrm{CP}$ contained $0.9 \% \mathrm{NaCl}$ saline solution.

\section{Product application}

Patients were asked to apply 2-3 pulverizations in each nostril, 3 to 4 times per day (morning, mid-day, evening, and before night rest). Patients were included in the study on day -6 , started treatment on day 1 and finished the treatment course on day 21 .

\section{Parameters recorded}

Patients were enrolled based on AR symptom severity scores (minimum 3 on a scale of 6), positive skin prick test for allergens and positive basophil count in the nasal smears ( $>10$ eosinophils/hpf).

Patients were asked to record the symptoms in the morning (AM) or evening (PM), just prior to dosing (instantaneous rating, [i]) and over the previous $12 \mathrm{~h}$ (reflective rating, [r]) for the nasal and ocular symptoms.

Daily scores of rhinorrhea, congestion, sneezing, and itching were recorded for each patient and mean values of these 4 parameters were calculated to obtain reflective Total Nasal Symptom Score (rTNSS) whereas three ocular symptoms (eye itching, tearing, and eye redness) were combined to calculate reflective Total Ocular Symptom Score (rTOSS). The instantaneous pre-dose morning ocular scores were summed to obtain an instantaneous pre-dose TOSS (am-pre-dose iTOSS). The scores of rhino-conjunctivitis quality of life were evaluated at the start of treatment (day 1 baseline) and at the end of treatment (day 21) on a 0 (excellent) to 20 (worst) rating scale using a standard RQLQ. The effects of activity related to working performance, sleep, other symptoms such as throat irritation or bad taste not related to nose or eye symptoms, practical problems such as difficulty to go out of home, nasal symptoms, eye symptoms and emotional effects were rated and the mean of individual values for each parameter was assessed to calculate RQLQ. Rescue medicine (RM) score was noted daily on a four-point scale $(0=$ no rhinitis medicine; $1=$ cetirizine, $10 \mathrm{mg} / \mathrm{d}$, $2=$ cetirizine, $20 \mathrm{mg} / \mathrm{d}, 3=$ systemic or topical corticosteroids for Allergic Rhinitis). The mean scores and number of patients in each group was evaluated.). When more than one RM was used on the same day, only the maximal score medication was recorded.

\section{Statistical analysis of results}

The proportion (\%) of patient with particular symptoms at baseline and end of study was reported. The demographic details were calculated using Wilcoxon rank sum test.

All the analyses were carried out on as "intent-to-treat" basis with Microsoft Excel and XLStat using the available data. Significant effects were those with a probability lower than $\alpha=0.05$. For each score, repeated measures analysis of variance (RMANOVA) determined differences in symptoms scores across study visits. Nemenyi post-hoc test provided pairwise comparisons in a group between baseline and the end of the study. Results were compared with baseline values (before $1^{\text {st }}$ treatment on day 1) in the same group and between the groups, at each time point.

\section{Results}

\section{Disposition of patients}

Although it was intended to obtain data on at least 18 patients in the $\mathrm{CP}$ group and 36 in the TP group, only 12 male (mean age 42.75 years) and 3 female (mean age 37.0 years) patients in the CP group and 26 male (mean age 39.8 years) and 5 female (mean age 42.8 years) patients completed the study (Figure $1 \&$ Table 1 ).

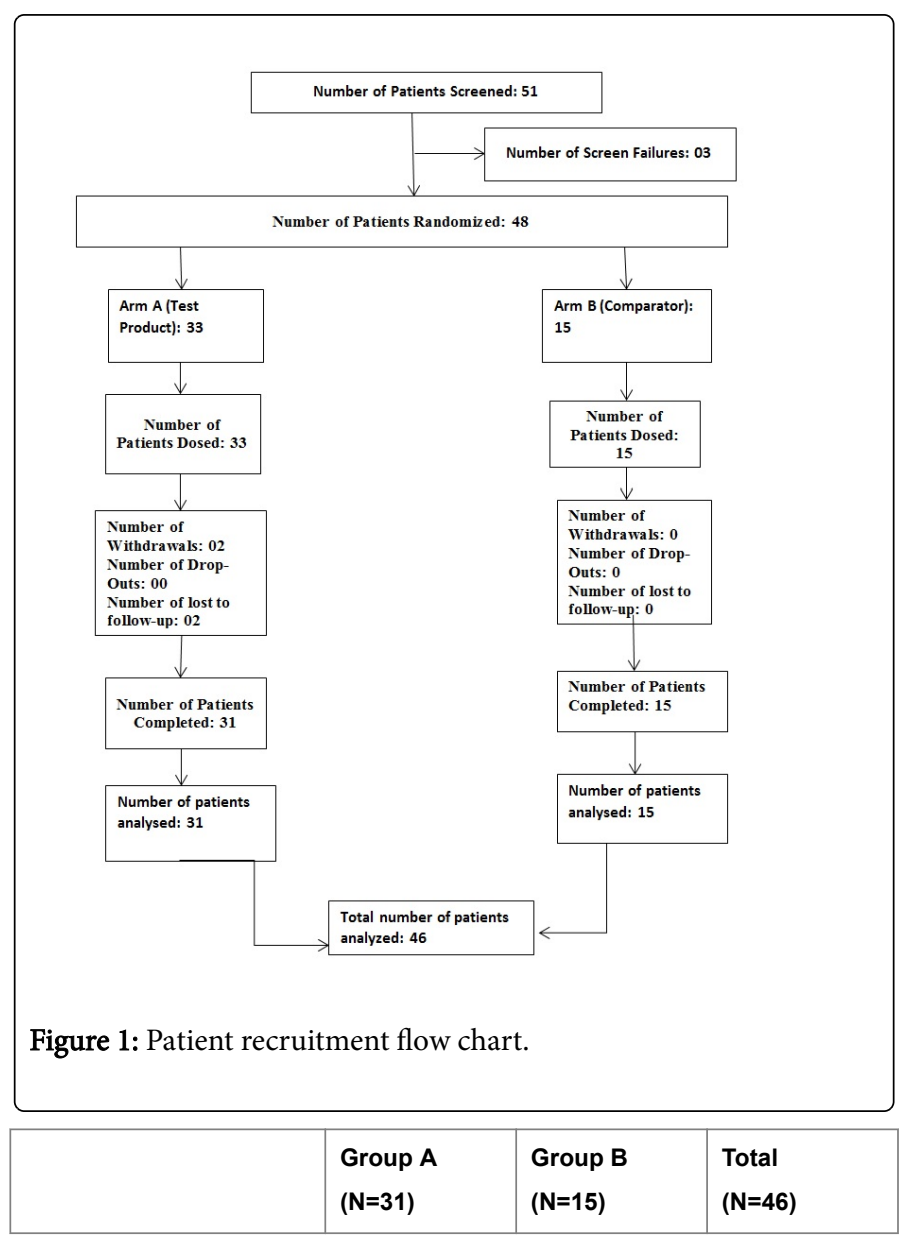




\begin{tabular}{|l|l|l|l|}
\hline Enrolled & 33 & 15 & 48 \\
\hline $\begin{array}{l}\text { Received at least one } \\
\text { dose }\end{array}$ & 33 & 15 & 48 \\
\hline $\begin{array}{l}\text { Received all doses and } \\
\text { attended all visits }\end{array}$ & 31 & 15 & 46 \\
\hline Completed & 31 & 15 & 46 \\
\hline $\begin{array}{l}\text { Withdrawn: } \\
\text { Lost to follow up }\end{array}$ & 02 & 00 & 02 \\
\cline { 2 - 5 } $\begin{array}{l}\text { Adverse event (No IP } \\
\text { effect) } \\
\text { Death } \\
\text { Other }\end{array}$ & 02 & 00 & 02 \\
\cline { 2 - 5 } & 00 & 00 & 12 \\
\hline
\end{tabular}

\begin{tabular}{|c|c|c|c|c|c|}
\hline & $\begin{array}{l}\text { Rhinorrh } \\
\text { ea }\end{array}$ & Congestion & Sneezing & Itching & $\begin{array}{l}M \\
e \\
a \\
n\end{array}$ \\
\hline Day -6 & 4.01 & 4.0 & 2.0 & 2.25 & 3.06 \\
\hline Week 0 & 4.98 & 4.48 & 3.27 & 3.99 & 4.18 \\
\hline Week 1 & 2.37 & 2.84 & 1.95 & 2.04 & 2.3 \\
\hline Week 2 & 1.72 & 1.8 & 1.15 & 1.33 & 1.5 \\
\hline Week 3 & 1.02 & 1.04 & 0.48 & 1.22 & 0.94 \\
\hline
\end{tabular}

Table 1: Patient enrollment and treatment profiles included in the test product (TP) group A and the control product $(\mathrm{CP})$ group $\mathrm{B}$ at the start of the study.

\section{Nasal symptoms scores (rTNSS)}

Only mean values are presented in this paper. Significant individual observations with respect to each symptom are mentioned in the discussion section. To compare differences in $\mathrm{CP}$ and $\mathrm{TP}$ groups at the start of treatment (T0), a compensation value, equal to the difference of mean score between these 2 groups at T0, is added to the mean of the lower baseline score group at all-time points. The following mean scores were obtained for rTNSS (Tables $2 \& 3$ ).

\begin{tabular}{|c|c|c|c|c|c|}
\hline \multicolumn{6}{|c|}{ Comparator Product } \\
\hline Period & Rhinorrhea & Congestion & Sneezing & Itching & $\begin{array}{l}\text { Mean } \\
\text { rTNSS }\end{array}$ \\
\hline Day -6 & 3.0 & 1.6 & 2.11 & 2.0 & 2.17 \\
\hline Baseline & 4.0 & 4.33 & 4.1 & 4.01 & 4.11 \\
\hline Week 1 & 3.66 & 3.44 & 3.71 & 3.67 & 3.62 \\
\hline Week 2 & 3.56 & 3.34 & 3.68 & 3.58 & 3.54 \\
\hline Week 3 & 3.5 & 3.29 & 3.6 & 3.53 & 3.48 \\
\hline \multicolumn{6}{|c|}{ Test Product } \\
\hline & \multicolumn{5}{|c|}{ Mean weekly rTNSS scores } \\
\hline
\end{tabular}

Table 2: Mean scores of Total Nasal Symptoms rTNSS in the comparator and test product groups at weekly intervals from the start (day -6) up to the week 3. rTNSS represents mean scores of rhinorrhea, nasal congestion, sneezing, and itching at each time point.

The baseline nasal symptoms of allergy rhinitis were relatively strong in both groups with slightly higher values in the TP group (4.18) compared to the CP group (4.11). To compare rTNSS mean scores, an adjustment value of 0.07 (4.18 - 4.11 in CP group) was added to all the $\mathrm{CP}$ mean values for unbiased comparison between the two groups. In this study, the initial recruitment period -6 day values show that, the nasal symptom intensity was strongly increased in both groups during the pre-treatment period. The mean values of rTNSS (Table 3) decreased strongly in TP during the $1^{\text {st }}$ week $(44.97 \%)$ and continued to diminish during week $2(64.11 \%)$ and week $3(77.51 \%)$ compared to baseline values $(\mathrm{p}<0.05)$. The same trend was observed when results are compared to those in the $\mathrm{CP}$ group. In this group, individual values show that rhinorrhea and nasal congestion were not affected during the $1^{\text {st }} 3$ days of treatment but decreased strongly thereafter up to the end of the study. It has been observed that the intensity of these two symptoms was high in TP group for about $30 \mathrm{~min}$ after each product application. This may have been related to the slight osmotic properties of TP. The individual values in CP group showed relatively important fluctuations during the study and the symptomatic relief observed usually lasted only for the $1^{\text {st }} 1-2 \mathrm{~h}$ after each product application. These improvements in CP group may have been due to the surface cleaning effects of the saline solution which could have minimized the concentration of histamines and allergenic particles in contact with the nasal mucosa. Results of rTNSS show that the mode of action of both $\mathrm{TP}$ and CP is closely identical but TP's effects are much faster and stronger.

\begin{tabular}{|c|c|c|c|c|}
\hline \multicolumn{5}{|c|}{ Study time-points } \\
\hline \multicolumn{5}{|l|}{ rTNSS } \\
\hline Day -6 & Baseline T0 & (Week 1) & (Week 2) & (Week 3) \\
\hline 2.17 & 4.11 & 3.62 & 3.54 & 3.48 \\
\hline \pm 0.68 & \pm 0.12 & \pm 0.54 & \pm 0.17 & \pm 0.36 \\
\hline 3.06 & 4.18 & 2.3 & 1.5 & 0.94 \\
\hline \pm 0.54 & \pm 0.18 & \pm 0.78 & \pm 0.70 & \pm 0.14 \\
\hline
\end{tabular}


Citation: Shrivastava L, Schütte H, Malik P, Shrivastava R (2017) A New Class of Polymeric Anti-Allergen Nasal Barrier Film Solution for the Treatment of Allergic Rhinitis. J Allergy Ther 8: 263. doi:10.4172/2155-6121.1000263

Page 5 of 9

\begin{tabular}{|c|c|c|c|c|c|}
\hline Adjustment required & & \multicolumn{4}{|c|}{ Compensation values +0.07} \\
\hline \multicolumn{6}{|l|}{ Adjusted values } \\
\hline Comparator group & & 4.18 & 3.69 & 3.61 & 3.55 \\
\hline Investigational group & & 4.18 & 2.3 & 1.5 & 0.94 \\
\hline \multicolumn{6}{|c|}{$\%$ Change versus T0 (baseline) } \\
\hline Comparator group & - & 0.0 & $-11.72 \%$ & $-13.63 \%$ & $-15.07 \%$ \\
\hline Investigational group & - & 0.0 & $-44.97 \%$ & $-64.11 \%$ & $-77.51 \%$ \\
\hline TP vs. CP & - & - & $-37.67 \%$ & $-58.44 \%$ & $-73.52 \%$ \\
\hline$P$ values & & & $\mathrm{P}<0.05$ & $P<0.05$ & $\mathrm{P}<0.05$ \\
\hline
\end{tabular}

Table 3: Mean absolute and compensated values of rTNSS in CP and TP groups on Day -6 and a weekly interval thereafter ( \pm SD) with statistical difference between TP vs. CP. A compensation value of 0.07 was added in CP group mean scores from the baseline up to week 3 to compare CP and TP groups identically from the start of treatment. Results are considered statistically significant compared to CP at the same time point if $\mathrm{p}<0.05$.

\section{Ocular symptoms scores (rTOSS)}

In the nasal-ocular reflex, allergens in the nose stimulate inflammatory mediators, which in turn stimulate the trigeminal ganglion leading to ocular vasodilatation, erythema, plasma leakage, and tearing.

The rTOSS scores (Table 4), particularly for eye tearing and eye redness were rather low in TP group compared to CP group at the time of recruitment (day -6) and even on the day of start of treatment (mean score 3.04/6 in TP group compared to 4.08/6 in CP group). The reason for this difference is not clear but may be due to enrollment of higher early AR stage patients in the TP group.

\begin{tabular}{|c|c|c|c|c|}
\hline \multicolumn{5}{|c|}{ Comparator CP group } \\
\hline & \multicolumn{3}{|l|}{ rToss } & \multirow[b]{3}{*}{ MEAN } \\
\hline & \multicolumn{3}{|c|}{ Means of Occular Symptoms } & \\
\hline & $\begin{array}{l}\text { Eye } \\
\text { Itching }\end{array}$ & Eye Tearing & Eye Redness & \\
\hline Day -6 & 3.44 & 3.6 & 3.98 & 3.67 \\
\hline Day 0 - Baseline & 4.06 & 3.86 & 4.33 & 4.08 \\
\hline Week 1 - Day 7 & 3.68 & 3.68 & 4 & 3.78 \\
\hline Week 2 - Day 14 & 3.58 & 3.5 & 3.86 & 3.64 \\
\hline Week 3 Day 21 & 3.58 & 3.42 & 3.8 & 3.6 \\
\hline \multicolumn{5}{|c|}{ Test product TP group } \\
\hline Day -6 & 2.31 & 1.7 & 1.67 & 1.893 \\
\hline Baseline-Day 0 & 3.12 & 3 & 3 & 3.04 \\
\hline Week 1-Day 7 & 1.78 & 1.47 & 1.82 & 1.69 \\
\hline Week 2-Day 14 & 1.17 & 1.11 & 1.26 & 1.18 \\
\hline
\end{tabular}

\begin{tabular}{|l|l|l|l|l|}
\hline Week 3-Day 21 & 1.02 & 0.83 & 0.91 & 0.92 \\
\hline
\end{tabular}

Table 4: Mean scores of Total Ocular Symptoms rTOSS in the comparator and test product groups at weekly intervals from the start (day -6) up to the week 3. rTOSS represents mean scores obtained for eye itching, tearing, and redness at each time point.

The mean rTOSS scores (Table 5) decreased slightly (10 to $15 \%$ compared to baseline) in the CP group but improvement was much stronger in the TP group as rTOSS mean score decreased by $38.3 \%$, $54.6 \%$, and $64.0 \%$ compared to $\mathrm{CP}$ at the end of Weeks 1, 2, and 3, respectively. Taking into consideration the reduction in rTNSS as well as in rTOSS, a potent effect of TP on the symptomatic manifestation of AR is observed.

\begin{tabular}{|c|c|c|c|c|}
\hline & \multicolumn{4}{|c|}{ Study time-points } \\
\hline & \multicolumn{4}{|l|}{ rTOSS } \\
\hline & T0: baseline & Week 1 & Week 2 & Week 3 \\
\hline \multicolumn{5}{|l|}{ Absolute values } \\
\hline Comparator group & 4.08 & 3.78 & 3.64 & 3.6 \\
\hline SD & \pm 0.23 & \pm 0.18 & \pm 0.18 & \pm 0.14 \\
\hline $\begin{array}{l}\text { Investigational } \\
\text { group }\end{array}$ & 3.04 & 1.69 & 1.18 & 0.92 \\
\hline SD & \pm 0.06 & \pm 0.28 & \pm 0.32 & \pm 0.10 \\
\hline $\begin{array}{l}\text { Adjustment } \\
\text { required : }\end{array}$ & \multicolumn{4}{|c|}{ Compensation values -1.04 in $\mathrm{CP}$} \\
\hline \multicolumn{5}{|l|}{ Adjusted values } \\
\hline Comparator group & 3.04 & 2.74 & 2.6 & 2.56 \\
\hline $\begin{array}{l}\text { Investigational } \\
\text { group }\end{array}$ & 3.04 & 1.69 & 1.18 & 0.92 \\
\hline \multicolumn{5}{|c|}{$\%$ Change versus T0 (baseline) and TP versus $\mathrm{CP}$ at each time point } \\
\hline Comparator group & - & $-9.86 \%$ & $-14.47 \%$ & $-15.78 \%$ \\
\hline
\end{tabular}


Page 6 of 9

\begin{tabular}{|l|l|l|l|l|}
\hline $\begin{array}{l}\text { Investigational } \\
\text { group }\end{array}$ & - & $-44.40 \%$ & $-61.18 \%$ & $-69.73 \%$ \\
\hline TP vs. CP & & $-38.32 \%$ & $-54.61 \%$ & $-64.06 \%$ \\
\hline p values & & $\mathrm{P}<0.05$ & $\mathrm{P}<0.05$ & $\mathrm{P}<0.05$ \\
\hline
\end{tabular}

\begin{tabular}{|l|l|l|l|l|}
\hline$\%$ TP vs. CP & & $-29.84 \%$ & $-48.91 \%$ & $-59.77 \%$ \\
\hline$p$ values & & 0.0005 & 0.0001 & 0.0001 \\
\hline
\end{tabular}

Table 6: Change in am-pre-dose Instantaneous total ocular symptom score (am-iTOSS) in CP and TP groups ( \pm SD) at baseline and at the end of the weeks 1,2 , and 3 . To compare the two-group score in an

Table 5: rTOSS mean scores and comparison at each time-point between $\mathrm{CP}$ and $\mathrm{TP}$ groups $( \pm \mathrm{SD})$ at baseline and at the end of the weeks 1,2 , and 3 . To compare the two-group score in an identical manner, an adjustment value (1.04) was deducted from CP scores at each time point to bring identical baseline scores in both groups at the start of treatment. The TP scores are then compared with baseline value and $v s . \mathrm{CP}$ as \% difference at each time point (statistically significant compared to $\mathrm{CP}$ if $\mathrm{p}<0.05$ ).

\section{AM pre-dose instantaneous total ocular symptoms scores (am-iTOSS)}

Mean results of iTOSS (Table 6 \& Figure 2) are comparable to that of rTOSS with a statistically significant reduction of ocular symptoms in the TP group compared to baseline or compared to $\mathrm{CP}$ group scores.

iTOSS: Being a main symptom of AR, the instantaneous pre-dose morning ocular scores were summed to obtain an instantaneous predose TOSS (AM pre-dose iTOSS). The baseline values in both CP and TP groups were nearly identical $(-0.09$ in $\mathrm{CP} v s$. TP) at the start of treatment. As shown in the Table 6 , the mean score was decreased only slightly and progressively in the CP group up to the end of the week 3 . In the TP group, the mean iTOSS score reduced very fast right after the start of treatment with $33 \%, 53 \%$, and $64 \%$ reduction at the end of the weeks 1,2 , and 3 compared to baseline values. Compared to CP, the decrease was nearly $30 \%, 49 \%$, and $59 \%$ at the same time points, indicating a strong effect on this parameter.

\begin{tabular}{|c|c|c|c|c|}
\hline & \multicolumn{4}{|c|}{ Study time-points } \\
\hline & \multicolumn{4}{|l|}{ iToss } \\
\hline & T0: baseline & Week 1 & Week 2 & Week 3 \\
\hline \multicolumn{5}{|l|}{ Absolute values } \\
\hline Comparator group & 2.10 & 2.00 & 1.93 & 1.88 \\
\hline SD & \pm 0.18 & \pm 0.62 & \pm 0.65 & \pm 0.64 \\
\hline $\begin{array}{l}\text { Investigational } \\
\text { group }\end{array}$ & 2.01 & 1.34 & 0.94 & 0.72 \\
\hline SD & \pm 0.12 & \pm 0.52 & \pm 0.48 & \pm 0.55 \\
\hline $\begin{array}{l}\text { Adjustment } \\
\text { required : }\end{array}$ & \multicolumn{4}{|c|}{ Compensation values -0.09 in $\mathrm{CP}$} \\
\hline \multicolumn{5}{|l|}{ Adjusted values } \\
\hline Comparator group & 2.01 & 1.91 & 1.84 & 1.79 \\
\hline $\begin{array}{l}\text { Investigational } \\
\text { group }\end{array}$ & 2.01 & 1.34 & 0.94 & 0.72 \\
\hline \multicolumn{5}{|c|}{$\begin{array}{l}\text { Pre-dose instantaneous total ocular symptoms (\% Change versus T0 } \\
\text { (baseline) }\end{array}$} \\
\hline Comparator group & - & $-4.97 \%$ & $-8.45 \%$ & $-10.94 \%$ \\
\hline $\begin{array}{l}\text { Investigational } \\
\text { group }\end{array}$ & - & $-33.33 \%$ & $-53.23 \%$ & $-64.17 \%$ \\
\hline
\end{tabular}
scores at each time point to bring identical baseline scores in both groups at the start of treatment. The TP scores are then compared with baseline value and vs. CP as \% difference at each time point (results are considered statistically significant compared to CP if $\mathrm{p}<0.05)$.

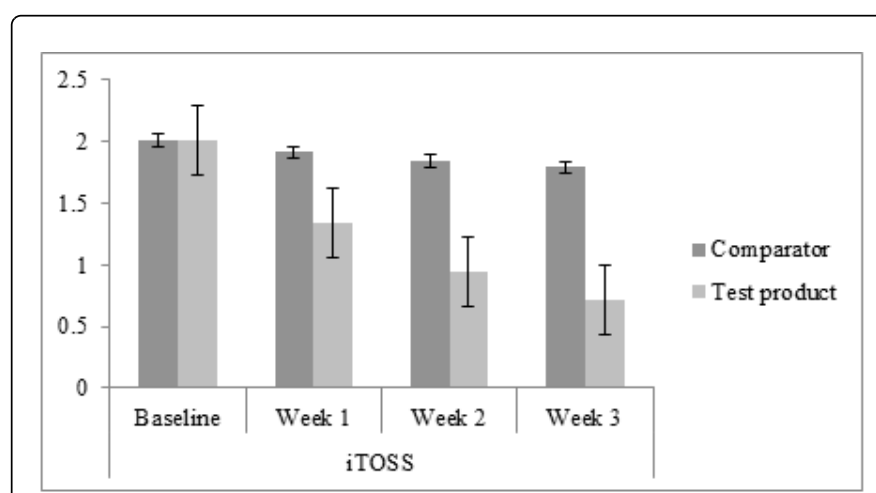

Figure 2: Mean iTOSS scores in $\mathrm{CP}$ and TP groups at baseline and at the end of the weeks 1,2 , and 3 ( \pm SD indicated in vertical bars). For TP vs. CP comparison, the baseline values were adjusted to the same level at baseline by adding an adjustment value $(-0.09)$ in the $\mathrm{CP}$ scores at each time point. Results are statistically significant compared to $\mathrm{CP}$ for the scores obtained at the end of the week 1,2 , and 3.

\section{Rhinoconjunctivitis quality of life (RQLQ)}

Changes in the mean of total score of Rhinoconjunctivitis Quality of Life (RQLQ) at the start of treatment (baseline) and at the end of treatment $( \pm$ SD) for TP and CP groups are shown in (Table 7).

\begin{tabular}{|c|c|c|c|c|}
\hline \multirow[t]{2}{*}{ RQLQ Domain } & \multicolumn{2}{|l|}{$\begin{array}{l}\text { Test group } \\
\mathrm{N}=31\end{array}$} & \multicolumn{2}{|l|}{$\begin{array}{l}\text { Placebo group } \\
\mathrm{N}=15\end{array}$} \\
\hline & Baseline & $\begin{array}{l}\text { End } \\
\text { study }\end{array}$ & Baseline & End of study \\
\hline & Mean $( \pm S D)$ & Mean $( \pm S D)$ & Mean $( \pm S D)$ & Mean $( \pm S D)$ \\
\hline Activity & $11.51( \pm 1.65)$ & $5.90( \pm 1.10)$ & $11.73( \pm 1.70)$ & $6.8( \pm 1.47)$ \\
\hline Sleep & $10.35( \pm 2.25)$ & $5.12( \pm 1.62)$ & $11.26( \pm 2.37)$ & $7.4( \pm 1.29)$ \\
\hline $\begin{array}{l}\text { Non-nose/non- } \\
\text { eye symptoms }\end{array}$ & $16.38( \pm 4.39)$ & $8.35( \pm 3.08)$ & $15.4( \pm 2.87)$ & $13.6( \pm 1.76)$ \\
\hline $\begin{array}{l}\text { Practical } \\
\text { problems }\end{array}$ & $10.29( \pm 1.88)$ & $5.38( \pm 1.89)$ & $12.6( \pm 2.12)$ & $6.8( \pm 1.65)$ \\
\hline $\begin{array}{l}\text { Nasal } \\
\text { symptoms }\end{array}$ & $12.09( \pm 2.03)$ & $5.96( \pm 1.70)$ & $9.86( \pm 2.06)$ & $\begin{array}{l}10.86 \\
2.47)\end{array}$ \\
\hline Eye symptoms & $12.35( \pm 2.21)$ & $5.09( \pm 2.13)$ & $11.6( \pm 1.63)$ & $9.93( \pm 1.94)$ \\
\hline
\end{tabular}




\begin{tabular}{|l|l|l|l|l|}
\hline Emotions & $12.38( \pm 2.85)$ & $6.64( \pm 1.90)$ & $17.2( \pm 2.07)$ & $13.8( \pm 1.65)$ \\
\hline Total & 85.35 & 42.44 & 89.65 & 69.19 \\
\hline $\begin{array}{l}\% \text { change vs. } \\
\text { baseline }\end{array}$ & $50.28 \%$ & & $22.85 \%$ \\
\hline
\end{tabular}

Table 7: The mean scores ( \pm SD) of 7 Rhinoconjunctivitis Quality of Life (RQLQ) parameters in TP and CP groups at the start of treatment (baseline) and at the end of the study (week 3). \% changes indicate \% difference between the total of mean scores of all the parameters at the end of the study compared to baseline value in the same group.

In the TP group, drastic reduction in the mean of all domains was seen while in placebo CP group, only mild to moderate reduction in all symptoms except nasal symptoms was noted.

There was mild increase in the nasal symptoms in the CP group. The mean scores per patient for 28 RQLQ questions at the start of treatment were $89.65( \pm 11.32)$ in the CP group and $85.35( \pm 10.55)$ in the TP group. At the end of the study (week 3 ), the mean score was $69.19( \pm 7.88)$ in the CP group $(-22.85 \%)$ versus $42.44(-50.28 \%, \pm$ $8.30)$ in the TP group. These results show a marked improvement of Quality of Life in the TP group compared to the CP group.

\section{Rescue medicine (RM) scores}

Total RM score was 29 for 15 patients in CP group and 23 for 31 patients in TP group. In TP group, $12 / 15$ patients (80\%) were given rescue medication at least once during the study period. 8 patients started taking RM on day $8 ; 2$ on day 15 , and another 2 on day 21 . In TP group, 9/31 patients (29\%) were administered RM, 8 on day 8 and 1 on day 15. TP patients used less RM compared to $\mathrm{CP}$ patients and for a shorter duration.

\section{Nasal eosinophil smears}

The mean eosinophil count in the CP group was 16.20 at baseline and decreased slightly to 13.72 at the end of week 3 (15.32\%). In TP group, the initial mean score of 16.16 at the start reduced drastically to 5.16 at the end of the study $(-68.07 \%)$. This drastic reduction in eosinophil count is an indication of reduced allergic activity on the surface of the nasal mucosa in TP patients which may be related to the filmogenic protection offered by the TP.

\section{Adverse events}

Total 12 patients had some adverse effects, 4 (26.7\%) in CP group and $8(25.8 \%)$ in TP group. These effects were reported at the end of week 2 (5 patients) and week 3 (8 patients) with complaints of headache ( 8 patients) and drowsiness ( 4 patients) which were not considered to be related to the administration of investigational products. No deaths or clinically significant changes in blood pressure, respiratory rate, or fever were recorded in either group, indicating that both comparator and test products are safe and act topically on the nasal surface.

\section{Discussion}

Rhinitis and rhinosinusitis, whether due to viral infections or to airborne allergens, are among the most common, non-life-threatening pathologies, in the world [13]. The first step towards treatment consists in identifying the origin of rhino-sinusitis, differentiating between infectious, allergic, non-allergic causes, and categorizing it as either chronic or acute [14].

Itching and ocular symptoms are particularly associated with allergic rhinitis, while other symptoms such as nasal congestion and obstruction, rhinorrhea, and sneezing may accompany both allergic and non-allergic rhinitis.

Diagnosis is usually confirmed by assessing patient history, reaction to contact with known allergens, seasonal history of rhinitis through skin prick test and detection of specific IgE antibodies in the patient's blood serum where treatment is usually administered as per standard guidelines published in each country $[15,16]$.

The first difficulty in treating rhinitis as reaction to common airborne allergens is to identify the causative allergens [17]. The second difficulty is to efficaciously prevent contact with that allergen, which can only be done when it is confined to a restricted area, otherwise exposure to allergens cannot be avoided. The third, harder to overcome, difficulty lies in late treatment, as almost all patients seek treatment only when symptoms have appeared and the immune cells of the nasal mucosa are already activated.

When allergens are presented to $\mathrm{T}$ and $\mathrm{B}$ lymphocytes, those secrete allergen-specific IgE antibodies that circulate in the peripheral blood stream, and attach to mast cells and basophils on the nasal mucosa $[18,19]$. Subsequent exposure to allergens activates these cells which instantly produce the classical inflammatory mediators of allergic reactions, including histamines [20]. As a local defense mechanism, the nasal mucosa then becomes totally infiltrated by inflammatory and immune cells, leading to a chronic inflammatory cascade and the typical symptoms of allergic rhino-sinusitis, which persist as long as new allergens come in contact with the nasal mucosa.

An ideal treatment should therefore not only stop, or prevent, the contact between the nasal mucosa and allergens, but should also minimize histamine-releasing and pro-inflammatory cells, as well as neutralize immune cell reactivity to the allergens, or simultaneously block the functions of all those cells. Such a treatment should also act only topically to avoid any generalized immune dysfunctions or adverse side effect, and to provide an ideal environment for the nasal mucosa to repair itself naturally.

Unfortunately, modern science has not made sufficient progress yet to act on multiple targets at once without creating serious immunological disorders in the body [21]. Current research is concentrated on finding specific inhibitors of undesirable proteins such as inhibitors of leukotriene and MMPs [22,23], but at present the use of topical or systemic antihistamines, corticoids, immunotherapy, nasal decongestants for blocked nose, or anticholinergics (e.g. ipratropium bromide) for runny nose, are the only symptomatic treatments available [24].

Our research was focused on finding a treatment that, if not ideal, would still be logical and multitargeted, against allergic rhinitis caused by common airborne allergens. This treatment is meant to not only prevent contact between allergens and the nasal mucosa, but also to remove the maximum number of immune cells from the surface of the nasal mucosa.

We envisaged using a highly filmogenic, non-irritant, cell-friendly, viscous solution, based on VB-Gy and film-forming polysaccharides, which, when applied onto biological tissue such as the nasal mucosa, can form a film over it, which would act as a protective barrier. 
As VB-Gy also possesses moderate osmotic properties, it can create an outward flow of hypotonic liquid from semi-permeable biological surfaces (e.g. nasal mucosa). As we did not want to exacerbate the rhinorrhea commonly present in allergic rhinitis, we selected a filmogenic yet not highly hypertonic solution, to privilege its filmforming characteristic over its osmotic activity. As the resulting solution nevertheless retained some slight osmotic capacity, it was hypothesized that the exudation would be mild and only sufficient to mechanically detach and remove the free-floating immune cells from the nasal surface along with histamine molecules, which would result in abatement of inflammation and dwindling of the inflammatory cascade [25].

The results of this study demonstrate that the $\mathrm{CP}$ is relatively active in minimizing the symptoms of AR as the mean scores of rTNSS, rTOSS, and iTOSS were decreased by nearly $15 \%$ while RQLQ was improved by $22 \%$. This is probably the reasons why saline solution nasal wash is still considered one of the best and the safest treatments against AR but nasal wash frequency must be high (every 3 to $4 \mathrm{~h}$ ) and regular to obtain symptomatic relief. The same results with TP were much faster and nearly 4-5 times prominent compared to CP indicating that TP's efficacy on AR is very strong. We observed, however, that due to the initially important nasal discharge, the film formed by VB-Gy solution becomes diluted more rapidly (within 15-30 $\mathrm{min}$ ) than desirable for a viable therapeutic option, as it does not offer long-lasting filmogenic barrier protection against incoming allergens as we expected. This is the reason why, just as polymers are used for enhancing flexibility and resistance of plastic materials [10], we incorporated specific polymers (Allercyanidins-H) of plant origin, capable of binding with VB-Gy molecules so as to improve the flexibility, resistance to liquid outflow, and duration of retention of the film over the live biological surface [26]. Even after being highly filmogen, TP film fade within 4-6 h, requiring 3-4 product applications per day to maintain nasal surface protection. During this period, new contact between the nasal mucosa and allergens is minimized, allowing the mucosa to repair itself and resume its normal defense functions. Due to slow outward exudation of hypotonic liquid, nasal mucosa also remains hydrated and clean, which is manifested in the form of reduction in rTNSS.

As polymer sources, we screened and selected some food grade plant and fruit extracts rich in polymeric tannins, the association of which was termed "Allercyanidin". Tannins are very large and branched, inert molecules that do not react with the nasal mucosa nor the cellular structures, and only play a role through their binding to the TP molecules [12]. All the TP ingredients has been extensively used for ages, orally as a food ingredient and topically in multiple cosmetic and pharmaceutical preparations, and are known to be totally safe. Glycerol is known to have no cellular interactions and this is the reason why diluted glycerol is used to preserve live cells and tissues while transport, to protect the cells while freezing in liquid nitrogen $\left(-196^{\circ} \mathrm{C}\right)$, and to store live tissue over many years [27]. The results of this study equally show that the VB-Gy-Allercyanidin film being flexible and preamble to water, hypotonic liquids and small molecules, it does not alter normal nasal mucosa functions.

The results of this clinical trial clearly show that regular use of VBGy-Allercyanidin- $\mathrm{H}$ filmogen spray bandage is highly effective in reducing symptomatic manifestations of allergic rhinitis, since all nasal and ocular symptoms of allergy decreased progressively yet remarkably in the TP group during the 3-week treatment period. Regular treatment with isotonic saline solution in the control group also produced some improvement in all allergic rhinitis symptoms, however reduction in severity was only about $15-20 \%$ compared to over $60-70 \%$ in the TP group. The relative, moderate efficacy of $0.9 \%$ $\mathrm{NaCl}$ saline wash is comprehensible as cleaning the nasal surface at regular, frequent intervals should drain away part of the allergens and immune cells, providing temporary, short-lasting and somewhat limited symptomatic relief.

It is not clear, from this study, why TP treatment produced progressive and marked alleviation of symptoms and why total recovery was not observed in all patients within 2-3 weeks. This may be due to the fact that the film protecting the nasal mucosa was not totally allergen-proof, or nasal mucosa functions may require longer than 3 weeks for complete resumption, or more simply, a much longer and continuous treatment may be required to offer more protection. The use of physical or mechanical nasal barriers to protect nasal mucosa against allergens is recent and lacks clinical data for comparison [28,29]. However, moderate clinical efficacy and total safety have been demonstrated with liposomal nasal barrier for the treatment of rhinosinusitis [30], glycerol esters [31] and atomized filmogen sprays [32] for the treatment of allergic rhinitis and glycerol polymer association for the treatment of cough [33]. This method of using a protective barrier against allergens was not explored much because in spite of being very safe, the major hurdle was keeping the barrier film intact over the nasal surface over sufficient period to allow nasal mucosa to resume its normal defensive functions. We rendered the film flexible and resistant to liquid flow, minimizing frequency of treatment and keeping the nasal mucosa physically away from allergens. Being a physical barrier, such a treatment presents an excellent safety profile and can be used by patients of any age and sex. Nevertheless, it should be kept in mind that such treatments shall remain a symptomatic, albeit efficacious, treatment, as immune cells conserve allergen memory over years or probably even decades, and will always react when exposed again to an identified allergen [34].

The primary downside of this trial is limited number of patients. It would have been better to have at least 60 patients in TP and 30 in CP including a few young $(<8$ years) patients and pregnant or lactating women. Similarly, comparing the TP efficacy directly against a reference drug, such as an antihistamine, would have given a better understanding of the product efficacy against a standard commonly used treatment. Nevertheless, this approach of covering the nasal mucosa with a flexible and resistant film like a bandage is found to be a highly promising, totally safe, mechanically acting, and entirely new generation of treatment against common allergic rhinitis. Further studies are warranted to evaluate the efficacy of this formula on other types of non-specific allergic rhinitis or on pollution-induced rhinosinusitis against reference drugs.

\section{Acknowledgments}

This clinical study was commissioned and funded by the Naturveda Laboratory.

\section{Conflict of Interest}

The authors have declared that no conflict of interest exists.

\section{References}

1. Berger WE (2006) Treatment of allergic rhinitis and other immunoglobulin E-mediated diseases with anti-immunoglobulin E antibody. Allergy Asthma Proc: 29-32. 
2. Skoner DP (2001) Allergic rhinitis: definition, epidemiology, pathophysiology, detection, and diagnosis. J Allergy Clin Immunol 108: 2-8.

3. Canonica GW, Bousquet J, Mullol J, Scadding GK, Virchow JC, et al. (2007) A survey of the burden of allergic rhinitis in Europe. Allergy 62: 17-25.

4. Small P, Harold K (2011) Allergic rhinitis. Allergy Asthma Clin Immunol 7:3-6.

5. Toppila-Salmi S, Drunen CMV, Fokkens WJ, Golebski K, Mattila P, et al. (2015) Molecular Mechanisms of Nasal Epithelium in Rhinitis and Rhinosinusitis. Curr Allergy Asthma Rep 15: 495-498.

6. Baraniuk JN (1997) Pathogenesis of allergic rhinitis. J Allergy Clin Immunol 99: 763-772.

7. Yang-G (2010) The pathophysiology, diagnosis and treatment of allergic rhinitis. Allergy Asthma Immunol Res 2: 65-76.

8. Holgate S, Casale T, Wenzel S, Bousquet J, Deniz Y, et al. (2005) The antiinflammatory effects of omalizumab confirm the central role of IgE in allergic inflammation. J Allergy Clin Immunol 115: 459-465.

9. Georges M, Ben Achour HM, Adly SA, Trouiller R, Shrivastava R, et al. (2017) Clinical Efficacy of a New Generation of multi-target, antiedematous, anti-inflammatory, tissue repairing topical polymeric liquid bandage for the treatment of internal hemorrhoids. J Clin Exp Dermatol Res 8: 403-412.

10. Roy A, Ghosh A, Datta S, Das S, Mohanraj P, et al. (2009) Effects of plasticizers and surfactants on the film forming properties of hydroxypropyl methylcellulose for the coating of diclofenac sodium tablets. Saudi Pharm J 17: 233-241.

11. Shrivastava RM, Shrivastava L, Shrivastava R (2013) Composition for topical application comprising glycerol and tannins. Patent PCT/ EP2013/061835, publication $\mathrm{N}^{\circ}$ WO2014194966 A1 dated 11th December 2014

12. Filanowicz M, Szynkiewicz E, Cegła B, Bartuzi Z (2016) Analysis of the quality of life of patients with asthma and allergic rhinitis after immunotherapy. Postepy Dermatol Alergol 33: 134-141.

13. Chad Z (2001) Allergies in children. Paediatr Child Health 6: 555-566.

14. Wallace DV, Dykewicz MS (2017) Seasonal allergic rhinitis: A focused systematic review and practice parameter update. Curr Opin Almlergy Clin Immunol 17: 286-294.

15. Kakli HA, Riley TD (2016) Allergic Rhinitis. Prim Care 43: 465-475.

16. Okubo K, Kurono Y, Ichimura K, Enomoto T, Okamoto Y, et al. (2017) Japanese guidelines for allergic rhinitis 2017. Allergol Int 66: 205-219.

17. Seidman MD, Gurgel RK, Lin SY, Schwartz SR, Baroody FM, et al. (2015) Clinical practice guideline: Allergic rhinitis. Otolaryngol Head Neck Surg 152: 1-43.

18. Quillen DM, Feller DB (2006) Diagnosing rhinitis: allergic vs nonallergic. Am Fam Physician 73: 1583-1590.
19. Siracusa MC, Kim BS, Spergel JM, Artis D (2013) Basophils and allergic inflammation. J Allergy Clin Immunol 132: 789-801.

20. Hellings PW, Klimek L, Cingi C, Agache I, Akdis C, et al. (2017) Nonallergic rhinitis: Position paper of the european academy of allergy and clinical immunology. Allergy.

21. Sin B, Togias A (2011) Pathophysiology of allergic and nonallergic rhinitis. Proc Am Thorac Soc 8: 106-114.

22. Tamada T, Ichinose M (2017) Leukotrine receptor antagonists and antiallergy drugs. Handb Exp Pharmacol 237: 153-169.

23. Pawankar R, Mori S, Ozu C, Kimura S (2011) Overview on the pathomechanisms of allergic rhinitis. Asia Pac Allergy 1: 157-167.

24. Cauwenberge P, Hoecke H (2005) Management of allergic rhinitis. BENT 1: 45-62.

25. Liang $\mathrm{M}, \mathrm{Xu} \mathrm{R}, \mathrm{Xu} \mathrm{G}$ (2015) Treatment strategies. [Recent advances in allergic rhinitis]. Lin Chung Er Bi Yan Hou Tou Jing Wai Ke Za Zhi 29: 202-206.

26. Shrivastava R, Rousse M, Dru L (2015) Recent Advances in the use of polymerics as topical protein antagonists. Eur J Pharm Med Res 2: 1627-1660.

27. Feilmeier MR, Tabin GC, Williams L, Oliva M (2010) The use of glycerolpreserved corneas in the developing world. Middle East Afr J Opthalmol; 17: 38-43.

28. AdeodatoVieira MG, Silva MA, Oliveirados L, Santo S, Beppu MM, et al. (2011) Natural-based plasticizers and biopolymer films: A review. European Polymer J 47: 254-263.

29. Payghan SA, Disouza JI. (2014) Fast dissolving HPMC E5 based oral film for rapid absorption of metoprolol tartrate. Eur J Pharma \& Medical Res 1: 75-91.

30. Eitenmüller A, Piano L, Böhm M, Shah-Hosseini K, Glowania A, et al. (2014) Liposomal nasal spray versus guideline-recommended steroid nasal spray in patients with chronic rhinosinusitis: A comparison of tolerability and quality of life. J Allergy (Cairo) 2014: 146280.

31. Ojeda P, Piqué N, Alonso A, Delgado J, Feo F, et al. (2013) A topical microemulsion for the prevention of allergic rhinitis symptoms: results of a randomized, controlled, double-blind, parallel group, multicentre, multinational clinical trial (Nares study). Allergy Asthma Clin Immunol 9:32.

32. Di Berardino F, Zanetti D, Amato G (2017) Nasal rinsing with an atomized spray improves mucociliary clearance and clinical symptoms during peak grass pollen season. Am J Rhinol Allergy 31: 40-43.

33. Shrivastava RM, Carrois F, Pisak M, Chabrillat T, Shrivastava R, et al (2017) Clinical Efficacy of a novel filmogen, antimicrobial, cleaning, fluidizing cough treatment. J Clin Trials 7: 318-326.

34. Wong KJ, Timbrell V, Xi Y, Upham JW, Collins AM, et al. (2015) IgE+ B cells are scarce, but allergen-specific B cells with a memory phenotype circulate in patients with allergic rhinitis. Allergy 70: 420-428. 